\title{
A new procedure for investigating three-dimensional stress fields in a thin plate with a through-the-thickness crack
}

\author{
Dake $\mathrm{Yi}^{i^{*}}$, and TzuChiang Wang ${ }^{2}$ \\ ${ }^{1}$ College of Mechanics and Materials, Hohai University, Nanjing 211100, China; \\ ${ }^{2}$ The State Key Laboratory of Nonlinear Mechanics, Institute of Mechanics, Chinese Academy of Sciences, Beijing 100190, China
}

Received August 14, 2017; accepted November 21, 2017; published online March 12, 2018

\begin{abstract}
In the paper, a new procedure is proposed to investigate three-dimensional fracture problems of a thin elastic plate with a long through-the-thickness crack under remote uniform tensile loading. The new procedure includes a new analytical method and high accurate finite element simulations. In the part of theoretical analysis, three-dimensional Maxwell stress functions are employed in order to derive three-dimensional crack tip fields. Based on the theoretical analysis, an equation which can describe the relationship among the three-dimensional $J$-integral $J(z)$, the stress intensity factor $K(z)$ and the tri-axial stress constraint level $T_{z}$ $(z)$ is derived first. In the part of finite element simulations, a fine mesh including 153360 elements is constructed to compute the stress field near the crack front, $J(z)$ and $T_{z}(z)$. Numerical results show that in the plane very close to the free surface, the $K$ field solution is still valid for in-plane stresses. Comparison with the numerical results shows that the analytical results are valid.
\end{abstract}

three-dimensional fracture analysis, out-of-plane constraint, three-dimensional $J$-integral

Citation: D. Yi, and T. C. Wang, A new procedure for investigating three-dimensional stress fields in a thin plate with a through-the-thickness crack, Sci. ChinaPhys. Mech. Astron. 61, 064611 (2018), https://doi.org/10.1007/s11433-017-9138-x

\section{Introduction}

Plate elements bearing tension, compression or bending are widely applied in a variety of engineering, such as aerospace engineering and civil engineering. The fracture analysis of plate elements undoubtedly is an important issue. In the past decades, many researchers have devoted their efforts to investigating the fracture problem of plate elements.

In earlier studies, considering a plate with a through-thethickness crack under pressure on the crack surfaces, Sih and Hartranft $[1,2]$ proposed an analytical method to examine the three-dimensional stress field near the crack front. A closed form solution, which depicts the variation of stress intensity factors along the plate thickness direction, was derived. The solution shows a boundary layer exists near the free surface

*Corresponding author (email: csruydk@163.com) of the plate. However, the closed form solution is very difficult to solve and equilibrium equations cannot be met in the boundary layer. Levy et al. [3] first applied finite element methods to investing the three-dimension stress field near the front of a crack in a thin plate. They concluded that a plane stress condition exists at a distance of twice the plate thickness away from the crack front along the mid-plane of the plate. Benthem [4] investigated the state of stress at the vertex of a quarter-infinite crack in a half space. In the paper, the stress tensor near the vertex is expressed in a separable form. In the vertex singular term, the leading root ranges from -0.5 to -0.332 when the Poisson's ratio varies from 0 to 0.5 . Benthem's solution can meet some numerical results from finite element methods [5]. However, Benthem's solution only partly satisfies boundary conditions at the free surface of the plate and might be verified further by more numerical results. Yang and Freund [6] studied the state of 
stress in a thin elastic plate which contains through-cracks. Based on the assumption that the through-the-thickness extensional strain was uniform in the thickness direction, these authors derived a crack tip boundary layer solution for the tensile opening mode of deformation (mode I). The solution shows a finite lateral contraction at the crack tip and the outof-plane displacement on the free surface of the plate merges smoothly with the corresponding plane stress solution at distances from the tip of one-half to three-fourths of the plate thickness. Using finite element methods, Nakamura and Parks [5] investigated the three-dimensional stress field near the front of a crack in a thin elastic plate. These authors revealed the variations of stress intensity factors, $J$-integrals and tri-axial stress constraint levels along the thickness direction. These authors also showed the numerical results of corner singular fields meet the solution given by Benthem at the free surface of the plate. However, the paper does not show the numerical results of corner singular fields in the boundary layer. Using 3-point bend test, Narasimhan and Rosakis [7] investigated three-dimensional effects near the crack front. They believed that plane stress conditions are reached at a distance of half the thickness from the crack front. Pook [8] reviewed the implications of corner point singularities. Pook concluded that a corner point singularity arises due to the influence of the Poisson's ratio and corner point singularities shall be taken into account in studying the problem of mixed mode fatigue crack growth. But, Pook also admitted that lack of appropriate numerical information makes it difficult to extract practical implications. Ranganathan et al. [9] showed experimental results on the characterization of the elastic-plastic strain fields at the crack tip due to cyclic loading. They presented isostrain contours and discussed the conditions of plane stress and plane strain. Su and Sun [10] carried out a three-dimensional stress asymptotic analysis for an elastic plate with a through-the-thickness crack under remote loading. They concluded that the stress singularity at the crack front is of inverse square root singularity throughout the plate thickness, and stress intensity factors can be expressed by a function of the thickness-wise coordinate. But, the function cannot be determined completely by the analysis method alone. Using global-local finite element methods, Kwon and Sun [11] performed threedimensional finite element analysis on an elastic plate with a through-the-thickness crack under remote loading. Based on their numerical results, Kwon and Sun proposed a $K-V$ interaction model to reveal the characterization of the threedimensional stress field around the crack front in the boundary layer. These authors also argued $\rho$ of the spherical region where the vertex singularity is dominant is less than $0.001 t$, where $\rho$ is a distance from the corner point and $t$ is the thickness of the plate. However, the paper focuses on discussing the numerical results in the symmetrical planes or at the free surface of the plate so the $K-V$ interaction model might be verified further by more numerical results of stress fields in the boundary layer. Based on the Kane and Mindlin theory as well as an application of the distributed dislocation technique, Kotousov [12] showed semi-analytical elastic results for crack tip opening displacement (CTOD) for a through-the-thickness crack in infinite plates with various thicknesses. Kotousov concluded that the transition from plane-strain to plane-stress conditions occurs when the ratio of crack length to plate thick is in the range of 0.01 to 10 . Using boundary layer and three-dimensional finite element methods, She and Guo [13] investigated three-dimensional distributions of stress near the front of a crack in a thin elastic plate under mixed-mode loading. Two empirical formula were fitted to describe the three-dimensional distribution of $T_{z}$ (the tri-axial stress constraint level) around the crack front for pure tension and pure shear cases. Employing three-dimensional finite element methods, Moreira et al. [14] calibrated the stress intensity factor in a cracked plate subjected to remote uniform traction. They concluded that the opening stress decreases quickly along the thickness direction in a boundary layer whose size is a function of the ratio of plate thickness to crack length. However, the expression of the function does not be given by these authors. Chaudhuri [15] developed an eigen-function expansion technique to derive three-dimensional asymptotic stress fields in the vicinity of the front of a semi-infinite through-crack weakening an infinite plate made of a homogeneous cubic single crystal. He concluded that when single crystals of alkali halides (rock salt structure) are subjected to tensile stress, no crack deviation is expected; in contrast, when single crystals of bcc metals are subjected to tensile stress, crack deflection always occurs. However, there are no experimental results to support these conclusions in the paper. Employing a threedimensional boundary-layer model for small-scale yielding, Sobotka and Dodds [16] explored $T$-stress effects on the three-dimensional, mode I crack-front fields for steady-state tearing in an elastic-plastic material. They revealed a strong effect of $T$-stress on key stress and strain quantities for low loading levels and a less effect for higher loading levels. But, the effect of out-of-plane constraint doesnot be considered in the paper. Kotousov et al. [17] investigated the effect of plate thickness on coupled stress intensity factors for an elastic plate with a through-the-thickness crack under shear or antiplane loading. These authors argued shear or anti-plane loading always generates coupled local fracture modes (Mode II+Mode III) due to Poisson's ratio effect and coupled stress intensity factors can be expressed by a power function of plate thickness. However, these authors also admitted the theoretical conclusions need further experimental confirmation. Using three-dimensional finite element methods and photo-elastic experimental methods, Khan et al. [18] investigated the effect of thickness on crack front core regions, stress intensity factors and crack initiation angles for 
an elastic plate with a through-the-thickness crack under mixed mode loading. They revealed that the plastic zone size near the crack front decreases with increasing thickness of the specimen both at the surface plane and the mid-plane. Using finite element methods, Góes et al. [19] examined three-dimensional effects on the stress/strain fields close to the tips of a notch and a long/short crack. They revealed that for a long crack, the size of the three-dimensional zone ahead of the crack tip is up to 0.4 times the plate thickness in the mid-plane, while for a short crack, the size of the threedimensional zone is up to 10 times the crack length. These authors also observed that peak values of stress intensity factors appear near the free surface for short cracks. However, these authors do not give any explanation for the phenomenon. Recently, He et al. [20] gave a brief review of recent three-dimensional studies of brittle fracture, especially the studies of the coupled fracture modes and threedimensional vertex singularities.

In the works mentioned above, there are a limited number of analytical solutions available in the literature because of mathematical complexities encountering in solving this kind of three-dimensional problems. In the current paper, a new procedure including a new analytical method and finite element simulations is proposed to investigate fracture problems of an elastic plate with a long through-the-thickness crack under mode-I loading. In the part of theoretical analysis, the main contribution is that a formula which depicts the relationship between $J$-integrals, stress intensity factors and tri-axial stress constraint levels is derived first. The formula reveal clearly the effect of tri-axial stress constraint level on stress intensity factor. In the part of numerical simulation, the first contribution is that the numerical results from finite element methods verify the solutions from the theoretical analysis. The second contribution is that numerical results show that the inverse square root singularity exists in the plane very close to the free surface of the plate.

\section{Physical backgrounds, basic equations and hypotheses for the current three-dimensional fracture problem}

\subsection{Physical backgrounds}

Consider a thin plate containing a through-the-thickness crack with thickness $2 \bar{h}$ subjected to remote uniform tensile loading $\bar{\sigma}^{\infty}$, as illustrated in Figure 1(a). It is well known that a thin plate without a crack under tension is in a state of plane stress. However, when the thin plate contains a through-thethickness crack under tensile loading, materials near the crack front contract in the $\bar{x}$ and $\bar{z}$ directions due to high stress normal to the crack plane, but these materials are prevented from doing so by the surrounding materials. This constraint causes a three-dimensional stress state near the crack front, as Figure 1(b) shows. Specially, at the mid-plane, the stress state is plane strain at distances from the crack front that are small compared to the plate thickness. Near the free surface, the through-the-thickness constraint is lower, and the out-of-plane stress vanishes at the free surface.

\subsection{Basic equations}

According to Hook's law, the stress-strain relations of elastic materials can be written as:

$\varepsilon_{i j}=(1+v) s_{i j}+\frac{1-2 v}{3} \sigma_{k k} \delta_{i j}$,

where, $v$ is Poisson's ratio and

$s_{i j}=\sigma_{i j}-\frac{1}{3} \sigma_{k k} \delta_{i j}$.

Throughout this paper, unless otherwise stated, all unbarred quantities are non-dimensional quantities. Length
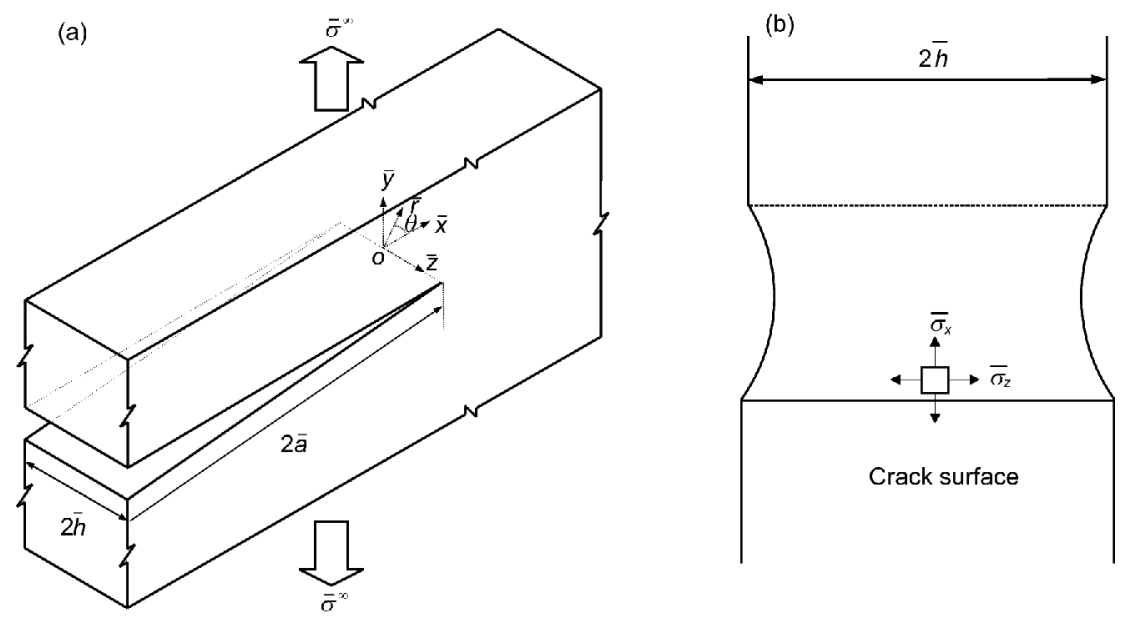

Figure 1 A thin plate containing a through-the-thickness crack subjected to remote uniform tensile loading $\bar{\sigma}^{\infty}$. (a) A cracked thin plate under tension; (b) a tri-axial stress state near the crack front. 
quantities if unbarred will be non-dimensionalized by the half thickness $\bar{h}$. Stress quantities if unbarred will be nondimensionalized by elastic modulus $\bar{E}$.

The equilibrium equations without body force can be expressed as:

$\sigma_{i j, j}=0$.

Employing non-dimensional Maxwell stress functions $\left(\phi_{i}\right.$, $i=1,2,3$ ), the stress components in the rectilinear coordinate system shown in Figure 2 may be expressed as:

$\sigma_{x}=\frac{\partial^{2} \phi_{2}}{\partial z^{2}}+\frac{\partial^{2} \phi_{3}}{\partial y^{2}}, \sigma_{y}=\frac{\partial^{2} \phi_{1}}{\partial z^{2}}+\frac{\partial^{2} \phi_{3}}{\partial x^{2}}$,

$\sigma_{z}=\frac{\partial^{2} \phi_{1}}{\partial y^{2}}+\frac{\partial^{2} \phi_{2}}{\partial x^{2}}, \sigma_{x y}=-\frac{\partial^{2} \phi_{3}}{\partial x \partial y}$,

$\sigma_{x z}=-\frac{\partial^{2} \phi_{2}}{\partial x \partial z}, \sigma_{y z}=-\frac{\partial^{2} \phi_{1}}{\partial y \partial z}$.

$\sigma_{r}=\cos ^{2} \theta \frac{\partial^{2} \phi_{2}}{\partial z^{2}}+\sin ^{2} \theta \frac{\partial^{2} \phi_{1}}{\partial z^{2}}+\frac{1}{r}\left(\phi_{3}\right)^{\prime}+\frac{1}{r^{2}}\left(\phi_{3}\right)^{\prime}$,

$\sigma_{\theta}=\sin ^{2} \theta \frac{\partial^{2} \phi_{2}}{\partial z^{2}}+\cos ^{2} \theta \frac{\partial^{2} \phi_{1}}{\partial z^{2}}+\left(\phi_{3}\right)^{\prime \prime}$,

$\sigma_{r \theta}=-\frac{\sin 2 \theta}{2}\left(\frac{\partial^{2} \phi_{2}}{\partial z^{2}}-\frac{\partial^{2} \phi_{1}}{\partial z^{2}}\right)+\frac{1}{r^{2}}\left(\phi_{3}\right)-\frac{1}{r}\left(\phi_{3}\right)^{\prime}$,

$\sigma_{z}=\sin ^{2} \theta\left(\phi_{1}\right)^{\prime \prime}+\frac{2 \sin \theta \cos \theta}{r}\left(\phi_{1}\right)^{\prime}+\frac{\cos ^{2} \theta}{r}\left(\phi_{1}\right)^{\prime}-\frac{2 \sin \theta \cos \theta}{r^{2}}\left(\phi_{1}\right)^{\prime}+\frac{\cos ^{2} \theta}{r^{2}}\left(\phi_{1}\right)$

$+\cos ^{2} \theta\left(\phi_{2}\right)^{\prime \prime}-\frac{2 \sin \theta \cos \theta}{r}\left(\phi_{2}\right)^{\prime}+\frac{\sin ^{2} \theta}{r}\left(\phi_{2}\right)^{\prime}+\frac{2 \sin \theta \cos \theta}{r^{2}}\left(\phi_{2}\right)^{\cdot}+\frac{\sin ^{2} \theta}{r^{2}}\left(\phi_{2}\right)^{\prime \prime}$,

$\sigma_{r z}=-\sin \theta\left(\sin \theta \frac{\partial\left(\phi_{1}\right)^{\prime}}{\partial z}+\frac{\cos \theta}{r} \frac{\partial\left(\phi_{1}\right)^{\prime}}{\partial z}\right)-\cos \theta\left(\cos \theta \frac{\partial\left(\phi_{2}\right)^{\prime}}{\partial z}-\frac{\sin \theta}{r} \frac{\partial\left(\phi_{2}\right)^{\prime}}{\partial z}\right)$,

$\sigma_{\theta z}=-\cos \theta\left(\sin \theta \frac{\partial\left(\phi_{1}\right)^{\prime}}{\partial z}+\frac{\cos \theta}{r} \frac{\partial\left(\phi_{1}\right)^{\prime}}{\partial z}\right)+\sin \theta\left(\cos \theta \frac{\partial\left(\phi_{2}\right)^{\prime}}{\partial z}-\frac{\sin \theta}{r} \frac{\partial\left(\phi_{2}\right)^{\prime}}{\partial z}\right)$,

where, ()$^{\prime}=\frac{\partial}{\partial \theta}$ and ()$^{\prime}=\frac{\partial}{\partial r}$.

The non-dimensional $\phi_{i}, r$ and $z$ are given by

$\phi_{i}=\frac{\overline{\phi_{i}}}{\bar{E}^{2}}, r=\frac{\bar{r}}{\bar{h}}, z=\frac{\bar{z}}{\bar{h}}$

It can be proved that eq. (5) satisfies the equilibrium equations in cylindrical coordinates, i.e.,

$\left(\sigma_{r}\right)^{\prime}+\frac{1}{r}\left(\sigma_{r \theta}\right)^{\prime}+\frac{\partial \sigma_{r z}}{\partial z}+\frac{\sigma_{r}-\sigma_{\theta}}{r}=0$,

$\left(\sigma_{r \theta}\right)^{\prime}+\frac{1}{r}\left(\sigma_{\theta}\right)^{\prime}+\frac{\partial \sigma_{\theta z}}{\partial z}+\frac{2 \sigma_{r \theta}}{r}=0$,

$\left(\sigma_{r z}\right)^{\prime}+\frac{1}{r}\left(\sigma_{\theta z}\right)+\frac{\partial \sigma_{z}}{\partial z}+\frac{\sigma_{r z}}{r}=0$.

An asymptotic expansion of the stress functions is at-

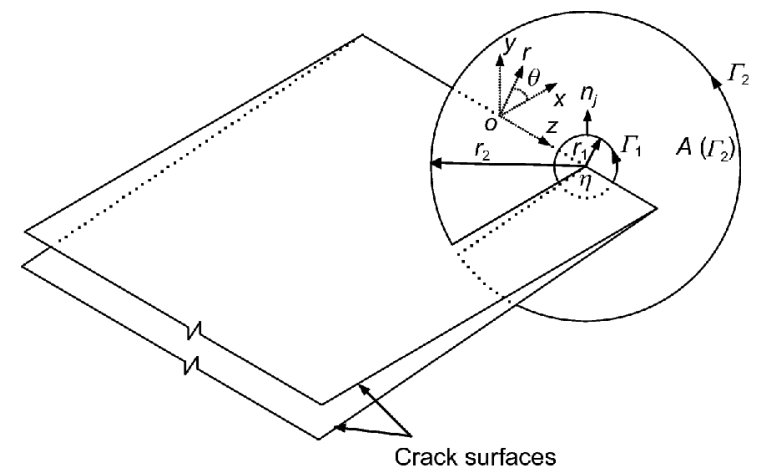

Figure 2 Three-dimensional $J$-integral.

Using the cylindrical coordinates $r, \theta$ and $z$ shown in Figure 2, eq. (4) may be re-written as: tempted in the form

$\phi_{i}=\sum_{n=1}^{\infty} F_{i}^{(n)}(z) r^{\frac{n+2}{2}} \widetilde{\phi}_{i}^{(n)}(\theta), \quad i=1,2,3$.

Our search will be restricted to only the dominant term in such an expansion:

$\phi_{1}=\phi_{2}=F_{1}(z) r^{\frac{3}{2}} \tilde{\phi}_{1}(\theta)$,

$\phi_{3}=\phi=F(z) r^{\frac{3}{2}} \tilde{\phi}(\theta)$.

Near the crack front, one may find

$\lim _{r \rightarrow 0}\left(\frac{\partial^{2} \phi_{i}}{\partial \mathbf{z}^{2}} \propto r^{\frac{3}{2}}\right)=0$,

$\lim _{r \rightarrow 0}\left(\frac{\partial^{2} \phi_{i}}{\partial z \partial r}, \frac{\partial \phi_{i}}{r \partial \theta} \propto r^{\frac{1}{2}}\right)=0$. 
Substituting eq. (9) into eq. (5) and noting eq. (10), the stress components may be re-written as:

$$
\begin{aligned}
\sigma_{r} & \cong \frac{1}{r}(\phi)^{\prime}+\frac{1}{r^{2}}(\phi)^{*}=F(z)\left(\frac{3}{2} \widetilde{\phi}+(\widetilde{\phi}) \cdot\right) r^{-\frac{1}{2},} \\
\sigma_{\theta} & \cong(\phi)^{\prime \prime}=\frac{3}{4} F(z) \tilde{\phi} r^{-\frac{1}{2}}, \\
\sigma_{r \theta} & \cong \frac{1}{r^{2}}(\phi)-\frac{1}{r}(\phi)^{\prime}=-\frac{1}{2} F(z)(\widetilde{\phi})^{-\frac{1}{2},} \\
\sigma_{z} & =\left(\phi_{1}\right)^{\prime \prime}+\frac{1}{r}\left(\phi_{1}\right)^{\prime}+\frac{1}{r^{2}}\left(\phi_{1}\right)^{\cdots} \\
& =F_{1}(z)\left(\frac{9}{4} \widetilde{\phi}_{1}+\left(\widetilde{\phi}_{1}\right)\right)^{\prime} r^{-\frac{1}{2}}, \\
\sigma_{r z} & \cong 0, \\
\sigma_{\theta z} & \cong 0 .
\end{aligned}
$$

Similarly, the stress components in the rectilinear coordinate system (eq. (4)) may be expressed as:

$$
\begin{aligned}
& \sigma_{x}=\frac{\partial^{2} \phi}{\partial y^{2}}, \sigma_{y}=\frac{\partial^{2} \phi}{\partial x^{2}}, \sigma_{z}=\frac{\partial^{2} \phi_{1}}{\partial x^{2}}+\frac{\partial^{2} \phi_{1}}{\partial y^{2}}, \\
& \sigma_{x y}=-\frac{\partial^{2} \phi}{\partial x \partial y}, \sigma_{x z}=0, \sigma_{y z}=0 .
\end{aligned}
$$

According to Hook's law, the strain components in the rectilinear coordinate system may be expressed as:

$\varepsilon_{x}=\frac{\partial^{2} \phi}{\partial y^{2}}-v\left(\frac{\partial^{2} \phi}{\partial x^{2}}+\frac{\partial^{2} \phi_{1}}{\partial x^{2}}+\frac{\partial^{2} \phi_{1}}{\partial y^{2}}\right)$

$\varepsilon_{y}=\frac{\partial^{2} \phi}{\partial x^{2}}-v\left(\frac{\partial^{2} \phi}{\partial y^{2}}+\frac{\partial^{2} \phi_{1}}{\partial x^{2}}+\frac{\partial^{2} \phi_{1}}{\partial y^{2}}\right)$

$\varepsilon_{z}=\frac{\partial^{2} \phi_{1}}{\partial x^{2}}+\frac{\partial^{2} \phi_{1}}{\partial y^{2}}-v\left(\frac{\partial^{2} \phi}{\partial x^{2}}+\frac{\partial^{2} \phi}{\partial y^{2}}\right)$

$\gamma_{x y}=-2(1+v) \frac{\partial^{2} \phi}{\partial x \partial y}, \gamma_{x z}=0, \gamma_{y z}=0$

The compatibility equations may be written as:

$$
\begin{aligned}
& \frac{\partial^{2} \varepsilon_{x}}{\partial y^{2}}+\frac{\partial^{2} \varepsilon_{y}}{\partial x^{2}}=\frac{\partial^{2} \gamma_{x y}}{\partial x \partial y}, \\
& \frac{\partial^{2} \varepsilon_{x}}{\partial z^{2}}+\frac{\partial^{2} \varepsilon_{z}}{\partial x^{2}}=\frac{\partial^{2} \gamma_{x z}}{\partial x \partial z}, \\
& \frac{\partial^{2} \varepsilon_{y}}{\partial z^{2}}+\frac{\partial^{2} \varepsilon_{z}}{\partial y^{2}}=\frac{\partial^{2} \gamma_{y z}}{\partial y \partial z} .
\end{aligned}
$$

Substituting the strain components (eq. (13)) to the compatibility equations (eq. (14)) one may obtain the partial differential governing equations

$\nabla^{4}(\phi)=0, \nabla^{4}\left(\phi_{1}\right)=0$,

$\nabla^{4}=\left(\frac{\partial^{2}}{\partial x^{2}}+\frac{\partial^{2}}{\partial y^{2}}\right)\left(\frac{\partial^{2}}{\partial x^{2}}+\frac{\partial^{2}}{\partial y^{2}}\right)$.

In the cylindrical coordinates, one may have

$$
\begin{aligned}
& \nabla^{4}(\phi)=0, \nabla^{4}\left(\phi_{1}\right)=0, \\
& \nabla^{4}=\left(\frac{\partial^{2}}{\partial r^{2}}+\frac{\partial}{r \partial r}+\frac{\partial^{2}}{r^{2} \partial \theta^{2}}\right)\left(\frac{\partial^{2}}{\partial r^{2}}+\frac{\partial}{r \partial r}+\frac{\partial^{2}}{r^{2} \partial \theta^{2}}\right) .
\end{aligned}
$$

\section{Solutions for three-dimensional singularity fields}

\subsection{Three-dimensional stress and strain fields}

Subsisting eq. (9) to eq. (16), one may have

$$
\begin{aligned}
& \tilde{\phi}^{\cdots \cdots}(\theta)+\frac{5}{2} \widetilde{\phi}^{\prime \prime}(\theta)+\frac{9}{16} \widetilde{\phi}(\theta)=0, \\
& \widetilde{\phi}_{1} \cdots(\theta)+\frac{5}{2} \widetilde{\phi}_{1} \cdot(\theta)+\frac{9}{16} \widetilde{\phi}_{1}(\theta)=0 .
\end{aligned}
$$

The general solutions for eq. (17) may be expressed as:

$\widetilde{\phi}(\theta)=A \cos \frac{\theta}{2}+B \sin \frac{\theta}{2}+C \cos \frac{3}{2} \theta+D \sin \frac{3}{2} \theta$,
$\widetilde{\phi}_{1}(\theta)=A_{1} \cos \frac{\theta}{2}+B_{1} \sin \frac{\theta}{2}+C_{1} \cos \frac{3}{2} \theta+D_{1} \sin \frac{3}{2} \theta$.

Boundary and symmetry conditions require

$\sigma_{\theta}(r, \pm \pi, z)=0$,

$\sigma_{r \theta}(r, 0, z)=0$,

$\sigma_{r \theta}(r, \pm \pi, z)=0$,

$\sigma_{z}(r, \theta, z)=\sigma_{z}(r,-\theta, z)$.

Then, eq. (18) may be re-written as:

$$
\begin{aligned}
& \tilde{\phi}=A\left(\cos \frac{1}{2} \theta+\frac{1}{3} \cos \frac{3}{2} \theta\right), \\
& \tilde{\phi}_{1}=A_{1} \cos \frac{1}{2} \theta+C_{1} \cos \frac{3}{2} \theta+D_{1} \sin \frac{3}{2} \theta .
\end{aligned}
$$

Substituting eq. (20) to eq. (11) and letting

$$
\begin{aligned}
& \frac{K(z)}{\sqrt{2 \pi}}=F(z) A, \\
& \frac{K_{1}(z)}{\sqrt{2 \pi}}=F_{1}(z) A_{1},
\end{aligned}
$$

one may have

$$
\begin{aligned}
& \sigma_{r}=\frac{K(z)}{\sqrt{2 \pi r}} \frac{1}{4}\left(5 \cos \frac{\theta}{2}-\cos \frac{3}{2} \theta\right), \\
& \sigma_{\theta}=\frac{K(z)}{\sqrt{2 \pi r}} \frac{1}{4}\left(3 \cos \frac{\theta}{2}+\cos \frac{3}{2} \theta\right), \\
& \sigma_{r \theta}=\frac{K(z)}{\sqrt{2 \pi r}} \frac{1}{4}\left(\sin \frac{\theta}{2}+\sin \frac{3}{2} \theta\right), \\
& \sigma_{z}=\frac{2 K_{1}(z)}{\sqrt{2 \pi r}} \cos \frac{\theta}{2}, \\
& \sigma_{r z} \cong 0, \\
& \sigma_{\theta z} \cong 0,
\end{aligned}
$$




$$
\begin{aligned}
& \sigma_{x}= \frac{\sigma_{r}+\sigma_{\theta}}{2}+\frac{\sigma_{r}-\sigma_{\theta}}{2} \cos 2 \theta-\sigma_{r \theta} \sin 2 \theta \\
&=\frac{K(z)}{\sqrt{2 \pi r}} \cos \frac{\theta}{2}\left(1-\sin \frac{1}{2} \theta \sin \frac{3}{2} \theta\right) \\
& \sigma_{y}= \frac{\sigma_{r}+\sigma_{\theta}}{2}-\frac{\sigma_{r}-\sigma_{\theta}}{2} \cos 2 \theta+\sigma_{r \theta} \sin 2 \theta \\
&=\frac{K(z)}{\sqrt{2 \pi r}} \cos \frac{\theta}{2}\left(1+\sin \frac{1}{2} \theta \sin \frac{3}{2} \theta\right), \\
& \tau_{x y}=\frac{\sigma_{r}-\sigma_{\theta}}{2} \sin 2 \theta+\sigma_{r \theta} \cos 2 \theta \\
&=\frac{K(z)}{\sqrt{2 \pi r}} \cos \frac{\theta}{2} \sin \frac{\theta}{2} \cos \frac{3}{2} \theta, \\
& \sigma_{z}=\frac{2 K_{1}(z)}{\sqrt{2 \pi r}} \cos \frac{\theta}{2}=\frac{2 T_{z}(z) K(z)}{\sqrt{2 \pi r}} \cos \frac{\theta}{2}, \\
& \sigma_{r z} \cong 0 \cong 0, \\
& \sigma_{\theta z} \cong 0
\end{aligned}
$$

where, $K(z)$ is the stress intensity factor (in dimensionless quantity). $T_{z}(z)$ is the tri-axial stress constraint coefficient defined as:

$T_{z}(z)=\frac{\sigma_{z}}{\sigma_{x}+\sigma_{y}}=\frac{K_{1}(z)}{K(z)}$.

Substituting eq. (23) to the stress-strain relations (eq. (1)), one may obtain strain components expressed as:

$$
\begin{aligned}
& \varepsilon_{\theta}=\frac{K(z)}{4 \sqrt{2 \pi r}}\left(\left(3-5 v-8 v T_{z}\right) \cos \frac{1}{2} \theta+(1+v) \cos \frac{3}{2} \theta\right), \\
& \varepsilon_{r}=\frac{K(z)}{4 \sqrt{2 \pi r}}\left(\left(5-3 v-8 v T_{z}\right) \cos \frac{1}{2} \theta-(1+v) \cos \frac{3}{2} \theta\right), \\
& \varepsilon_{r \theta}=\frac{K(z)}{4 \sqrt{2 \pi r}}(1+v)\left(\sin \frac{1}{2} \theta+\sin \frac{3}{2} \theta\right), \\
& \varepsilon_{z}=\frac{2 K(z)}{\sqrt{2 \pi r}}\left(T_{z}-v\right) \cos \frac{1}{2} \theta, \\
& \gamma_{z r}=0 \\
& \gamma_{z \theta}=0 .
\end{aligned}
$$

\subsection{Relationships among three-dimensional $J$-integral $J(z), K(z)$ and $T_{z}(z)$}

A mathematical expression of the three-dimensional $J$-integral (in dimensionless quantity) [21-23] is

$$
\begin{aligned}
& J(\eta)=\lim _{r_{1} \rightarrow 0} \int_{\Gamma_{1}}\left(W \mathrm{~d} y-\sigma_{i j} n_{j} u_{i, x} \mathrm{~d} s\right) \\
& \quad=\int_{\Gamma_{2}}\left(W \mathrm{~d} y-\sigma_{i j} n_{j} u_{i, x} \mathrm{~d} s\right)-\int_{A\left(\Gamma_{2}\right)} \frac{\partial\left(\sigma_{i z} u_{i, x}\right)}{\partial z} \mathrm{~d} A\left(\Gamma_{2}\right), \\
& i=x, y, z, j=x, y,
\end{aligned}
$$

where, $\eta$ is a point along the crack front, $J(\eta)$ is the pointwise value of the $J$-integral at a given point $\eta$ along the crack front (Figure 2), $W$ is the strain energy density (in dimensionless quantity), $\sigma_{i j}$ and $u_{i}$ are the Cartesian components of stress and displacement. Integral paths $\Gamma_{1}$ (radius $r_{1}$ ) and $\Gamma_{2}$ (radius $r_{2}$ ) lie in the plane perpendicular to the crack front, and $n_{j}$ are the components of a unit vector outward normal to the integral paths and normal to the crack front. $A\left(\Gamma_{2}\right)$ is the domain bounded by $\Gamma_{2}$.

The integral of strain energy density $W$ along the path $\Gamma_{1}$ may be expressed as:

$\int_{\Gamma_{1}} W \mathrm{~d} y=\int_{-\pi}^{\pi} W r_{1} \cos \theta \mathrm{d} \theta$

$\cong \int_{-\pi}^{\pi} \frac{1}{2}\left(\sigma_{r} \varepsilon_{r}+\sigma_{\theta} \varepsilon_{\theta}+\sigma_{z} \varepsilon_{z}+2 \sigma_{r \theta} \varepsilon_{r \theta}\right) r_{1} \cos \theta \mathrm{d} \theta$.

Substituting the stress components in eq. (22) and the strain components in eq. (25) to eq. (27), one may obtain

$$
\int_{\Gamma_{1}} W \mathrm{~d} y=\frac{K^{2}}{4}\left(2\left(T_{z}\right)^{2}-4 v T_{z}-v+1\right) .
$$

Similarly, the second integral in the first line in eq. (26) may be expressed as:

$$
\begin{aligned}
& -\int_{\Gamma_{1}} \sigma_{i j} n_{j} u_{i, x} \mathrm{~d} s \\
& =-\int_{-\pi}^{\pi}\left(\sigma_{x} n_{x} u_{x, x}+\sigma_{x y} n_{y} u_{x, x}+\sigma_{x y} n_{x} u_{y, x}+\sigma_{y} n_{y} u_{y, x}\right) r_{1} \mathrm{~d} \theta \\
& =-\int_{-\pi}^{\pi}\left[\sigma_{r}\left(-\frac{\sin \theta}{r_{1}}\left(\left(u_{r}\right)-\left(u_{\theta}\right)\right)+\left(u_{r}\right) \cos \theta\right)\right. \\
& \left.+\sigma_{r \theta}\left(-\frac{\sin \theta}{r_{1}}\left(\left(u_{\theta}\right)+u_{r}\right)+\cos \theta\left(u_{\theta}\right)\right)\right] r_{1} \mathrm{~d} \theta \\
& =-\frac{K^{2}}{4}\left(2 v T_{z}-v-3\right) .
\end{aligned}
$$

Here, according to the strain-displacement relations, $u_{r}$, $\left(u_{r}\right),\left(u_{\theta}\right)$ and $u_{\theta}$ may be expressed by

$$
\begin{aligned}
& u_{r}=\int \varepsilon_{r} \mathrm{~d} r=\frac{K \sqrt{r}}{2 \sqrt{2 \pi}}\left(\left(5-3 v-8 v T_{z}\right) \cos \frac{1}{2} \theta-(1+v) \cos \frac{3}{2} \theta\right), \\
& \left(u_{r}\right)=\frac{K \sqrt{r}}{4 \sqrt{2 \pi}}\left(-\left(5-3 v-8 v T_{z}\right) \sin \frac{1}{2} \theta+3(1+v) \sin \frac{3}{2} \theta\right), \\
& \left(u_{\theta}\right)^{\cdot}=\varepsilon_{\theta} r-u_{r} \\
& =\frac{K \sqrt{r}}{4 \sqrt{2 \pi}}\left(\left(-7+v+8 v T_{z}\right) \cos \frac{1}{2} \theta+3(1+v) \cos \frac{3}{2} \theta\right), \\
& u_{\theta}=\frac{K \sqrt{r}}{2 \sqrt{2 \pi}}\left(\left(-7+v+8 v T_{z}\right) \sin \frac{1}{2} \theta+(1+v) \sin \frac{3}{2} \theta\right) \text {. }
\end{aligned}
$$

Derivations of eqs. (28) and (29) have been shown in the Appendix.

Substituting eqs. (28) and (29) to the expression of the three-dimensional $J$-integral (eq. (26)), one gets the relationships among $J(z), K(z)$ and $T_{z}(z)$, expressed as:

$$
\left\{\begin{aligned}
J(z) & =\int_{\Gamma_{1}}\left(W \mathrm{~d} y-\sigma_{i j} n_{j} u_{i, x} \mathrm{~d} s\right) \\
& =\frac{K^{2}(z)}{2}\left(\left(T_{z}(z)\right)^{2}-3 v T_{z}(z)+2\right), \\
\text { or } & \\
K(z) & =\sqrt{\frac{2 J(z)}{\left(T_{z}(z)\right)^{2}-3 v T_{z}(z)+2}},
\end{aligned}\right.
$$


specially, $J=K^{2}$ when $T_{z}=0$ (plane stress) and $J=\left(1-v^{2}\right) K^{2}$ when $T_{z}=v$ (plane strain).

\subsection{Three dimensional finite element mesh}

$K(z)$ is undetermined and should be related to the applied stress. On the other hand, eq. (31) shows $K(z)$ is a function of $J(z)$ and $T_{z}(z)$. If the values of $J(z)$ and $T_{z}(z)$ can be obtained using three-dimensional finite element methods, the value of $K(z)$ can be resolved by eq. (31) and the stress components near the crack front can be calculated based on eqs. (22) and (23).

A circular disk (cylinder) is used to model the near tip region of a thin plate with thickness $2 \bar{h}$, as shown in Figure 3(a). Only a quarter of circular disk (region $0 \leq \theta \leq \pi$, $0 \leq \bar{z} / \bar{h} \leq 1)$ is modeled with finite elements since the symmetry of the current problem, as shown in Figure 3(b). The finite element mesh is constructed with 8-node brick elements. In the plane perpendicular to the crack front $\left(\bar{x}^{-} \bar{y}\right.$ plane), the element size is gradually increased with radial distance from the crack front (the progression ratio is 1.21), while the angular increment of each element is kept constant, $\Delta \theta=\frac{\pi}{36}$. The identical planar mesh is repeated along the $z$ axis from the symmetry-plane $(z=0)$ to the free-surface $(z=1)$. The thickness of successive element layers gradually increases towards the symmetry plane $(z=0)$ (the progression ratio is 1.12). The half thickness of the plate consists of 60 layers, and the thickness of the layer at the free surface is $1.11 \times 10^{-4} \bar{h}$. The radial size of the elements around the crack front is $2 \times 10^{-5} \bar{h}$. There are 153360 elements in the current mesh. The mesh has been shown in Figure 3(b).

The symmetry conditions require:

(1) the normal displacements on the surface where $\bar{y}=0$ and $\bar{x} \geq 0$ should be zero,

(2) and the normal displacements on the symmetry plane where $\bar{z}=0$ should be zero.

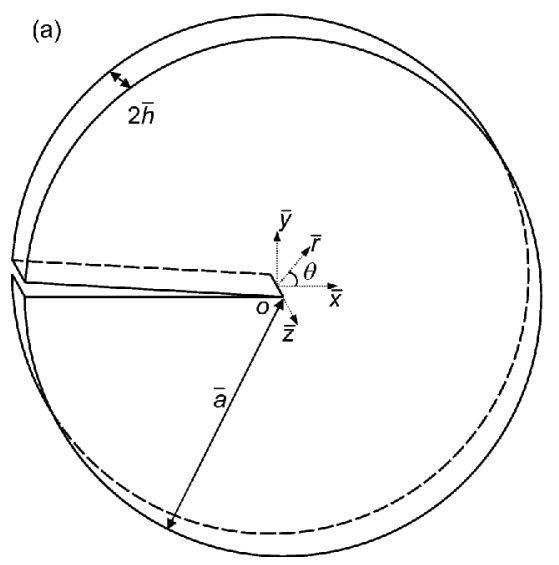

The stress distribution on the surface where $r=a$ $(a=\bar{a} / \bar{h}=10)$ is given by

$$
\begin{aligned}
& \sigma_{r}=\frac{K^{\text {far }}}{\sqrt{2 \pi a}} \frac{1}{4}\left(5 \cos \frac{\theta}{2}-\cos \frac{3}{2} \theta\right), \\
& \sigma_{r \theta}=\frac{K^{\text {far }}}{\sqrt{2 \pi a}} \frac{1}{4}\left(\sin \frac{\theta}{2}+\sin \frac{3}{2} \theta\right), \\
& \sigma_{r z}=0 .
\end{aligned}
$$

\section{Results and discussion}

Figures 4-6 show that the values of $J(z), T_{z}(z)$ and $K(z)$ decrease from the mid-plane $(z=0)$ to the plane very near the surface $(z=0.995)$. Figure 4 shows that all of the curves pass through (near) a point located at $z=0.6$ and $\frac{J}{\left(K^{\text {far }}\right)^{2}}=1$. The value of $J(z)$ for $v=0$ is kept constant and equals the corresponding two-dimensional solution. In Figure 5, one may observe that if $v \neq 0$, the values of $T_{z}(z)$ are high near the crack front at the mid-plane, so a plane strain condition may be met approximately. If $v=0, T_{z}(z)$ vanishes and the stress state near the crack front is plane stress. Figure 6 shows that all of the curves pass through (near) a point located at $z=0.94$ and $\frac{K}{K^{\text {far }}}=1$. The value of $K(z)$ for $v=0$ is kept constant and equals the corresponding two-dimensional solution. Figure 6 also shows the value of $K(z)$ for $v \neq 0$ drops quickly in the region $0.94 \leq z \leq 0.995$. The numerical results of nonzero stress components near the crack front at mid-plane and corresponding analytical results are plotted in Figure 7. The analytical results are in good agreement with the numerical results. Figure 8 shows that the numerical results of in-plane stress components near the crack front agree well with analytical results in the plane which is very close to the free surface. These results in Figure 8 imply that the singularity of in-plane stress components are still the inverse square root in

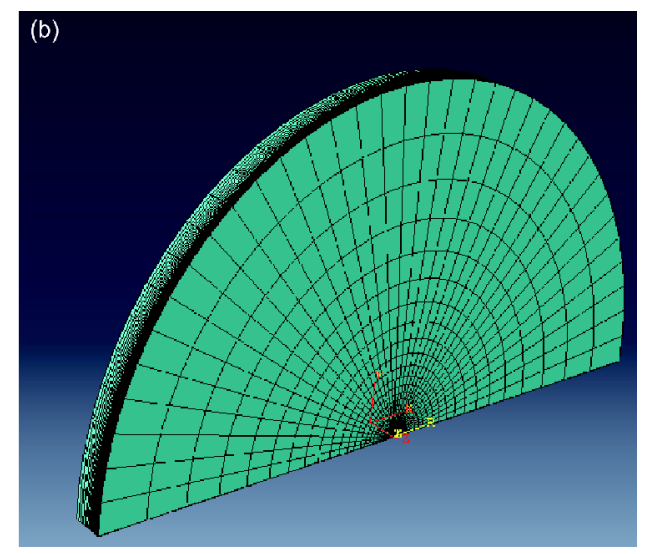

Figure 3 (Color online) (a) Schematic of a cracked circular disk which represents the near crack front region of a thin plate. Here, $\bar{a}=10 \bar{h}$. (b) Finite element mesh of the quarter-mode. 

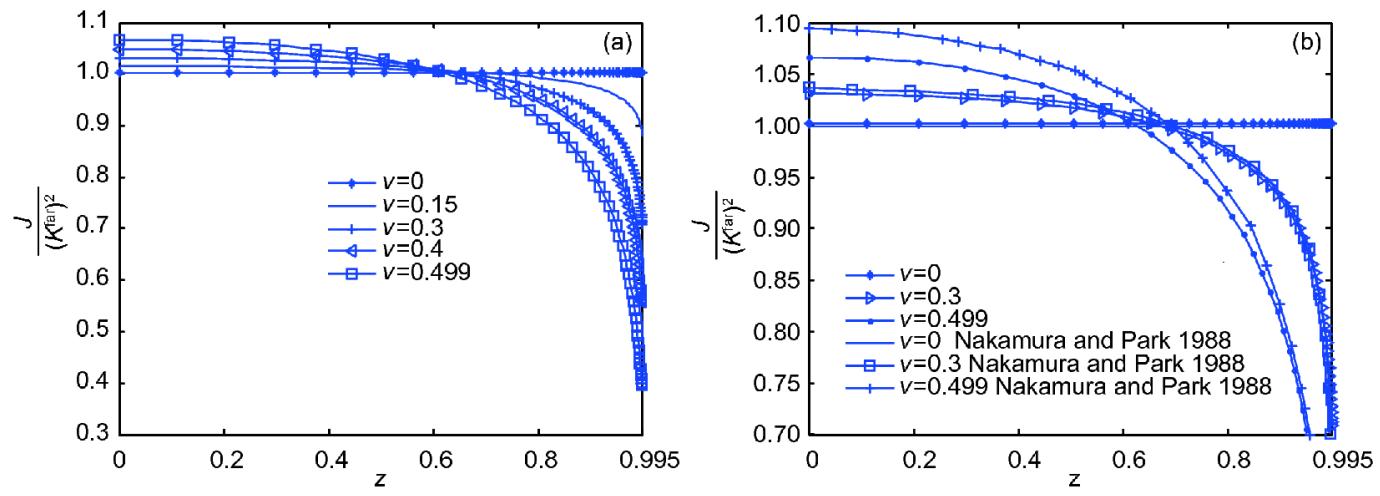

Figure 4 (Color online) (a) $\frac{J}{\left(K^{\text {far }}\right)^{2}}$ along the half-crack front for various Poisson's ratios; (b) comparison with Nakamura and Park's results.

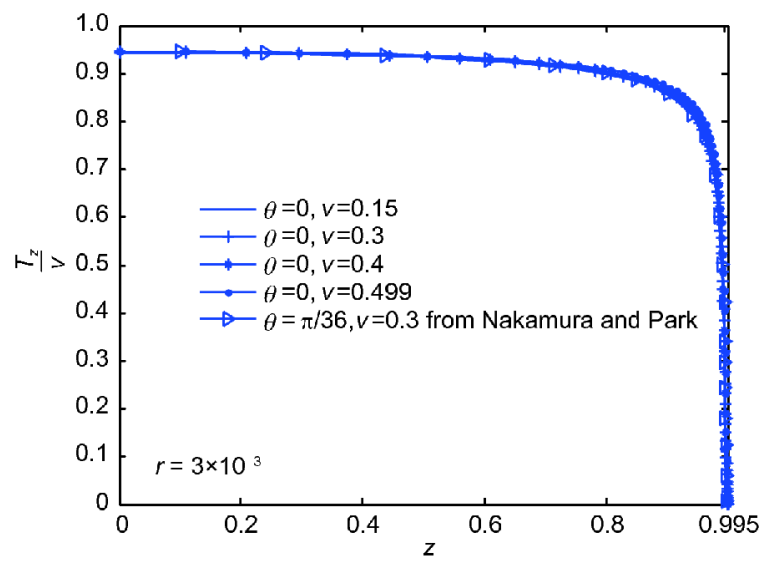

Figure 5 (Color online) $\frac{T_{z}}{v}$ along the half-crack front for various Poisson's ratios.

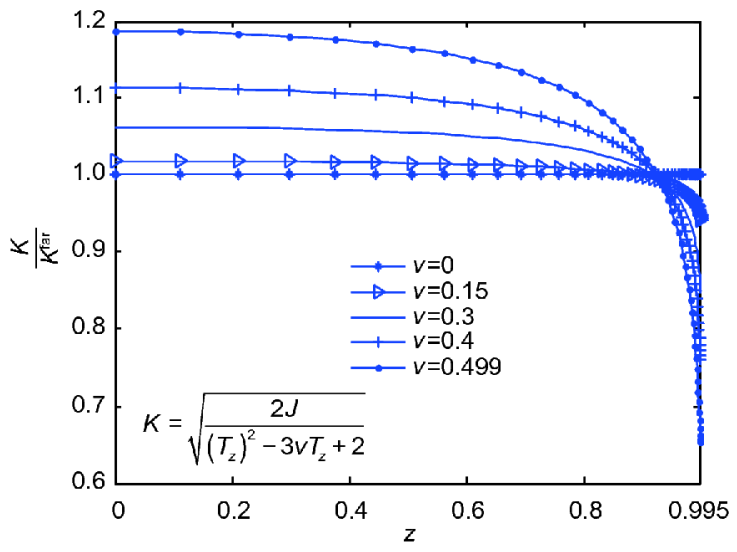
Figure 6 (Color online) $\frac{K}{K^{\text {far }}}$ along the half-crack front for various
Poisson's ratios.

the plane very close to the free surface. Figure 8 also shows that in the plane perpendicular to the crack front and very close to the free surface, the radium of the region where the $K$-field solution is dominant decreases with increasing $z$. For example, in the case that $v$ is 0.3 , when $z$ increases from 0.98 to 0.995 , the radium decreases from $3 \times 10^{-3}$ to $10^{-3}$. Figure 8 also implies eq. (31) derived by the current analytical method is valid in the case of lower tri-axial stress constraint level.

\section{Summary and conclusion}

In the current paper, a new procedure including theoretical analysis and high accurate finite element simulations is proposed to investigate the three-dimensional fracture problem of a thin elastic plate with a long through-the-thickness crack under remote tension. In the part of theoretical analysis, Maxwell stress functions are employed to derive the crack front singularity field. A formula (eq. (31)) is derived first, with aid of Figure 9 to calculate the three-dimensional stress intensity factor $K(z)$ after the three-dimensional $J$ integral $J(z)$ and the tri-axial stress constraint level $T_{z}(z)$ have been obtained by numerical methods. In the part of finite element simulations, a fine mesh including 153360 elements is constructed to compute the stress field near the crack front, $J(z)$ and $T_{z}(z)$. Comparison with the numerical results shows that the analytical results are valid. This work supports the following observations and conclusions.

(1) When $v=0$, the stress state near the crack front is plane stress.

(2) The three-dimensional stress intensity factor is a function of the three-dimensional $J$-integral $J(z)$ and the triaxial stress constraint level $T_{z}(z)$ and may be expressed as:

$K(z)=\sqrt{\frac{2 J(z)}{\left(T_{z}(z)\right)^{2}-3 v T_{z}(z)+2}}$.

(3) In the plane very close to the free surface, e.g., $z=0.995$ and $v=0.3$, the $K$ field solution is still valid for in-plane stresses.

(4) In the plane perpendicular to the crack front and close to the free surface, the radium of the region where the $K$-field solution is dominant decreases with increasing $z$. For example, in the case that $v$ is 0.3 , when $z$ increases from 0.98 to 0.995 , the radium decreases from $3 \times 10^{-3}$ to $10^{-3}$. 

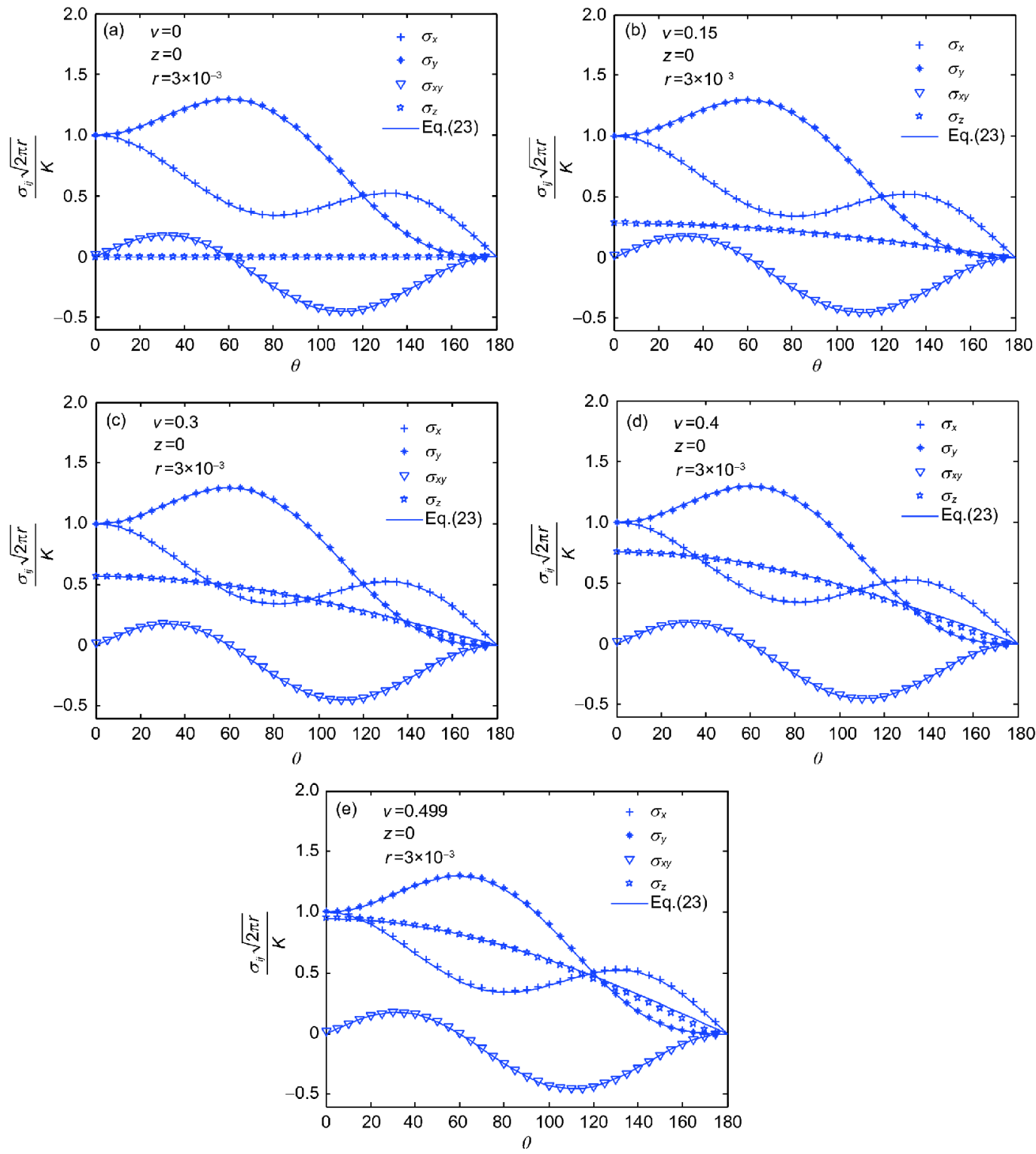

Figure 7 (Color online) Angular distributions of nonzero stress components normalized by $K$ near the crack front at mid-plane. Here $K=\sqrt{\frac{2 J}{\left(T_{z}\right)^{2}-3 v T_{z}+2}}$
(a) $v=0$, (b) $v=0.15$, (c) $v=0.3$, (d) $v=0.4$, and (e) $v=0.499$.
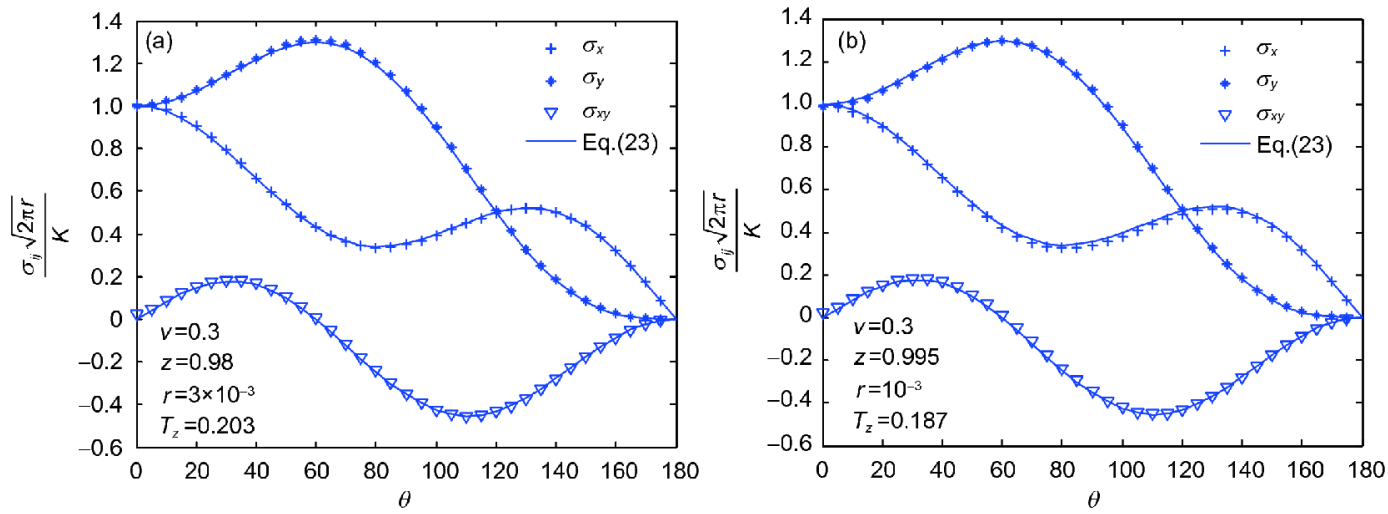

Figure 8 (Color online) Angular distributions of in-plane stress components normalized by $K$ near the crack front in the plane very close to the free surface. Here $K=\sqrt{\frac{2 J}{\left(T_{z}\right)^{2}-3 v T_{z}+2}}$. (a) $v=0.3, z=0.98$, (b) $v=0.3, z=0.995$. 


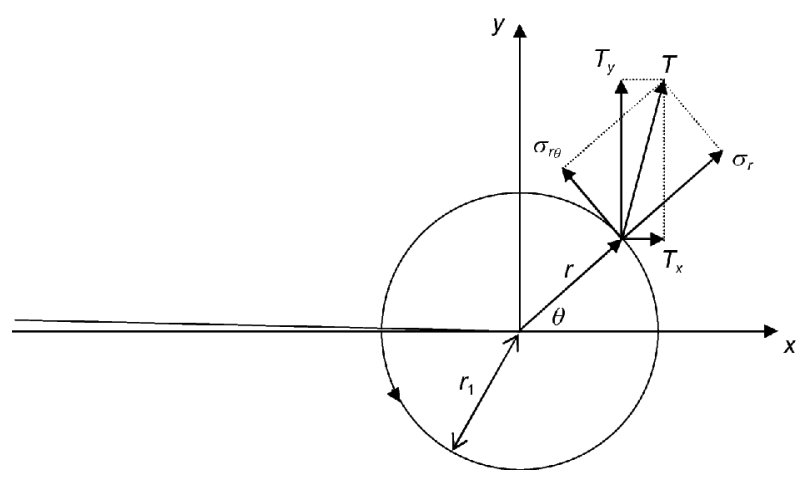

Figure 9 Traction $T$ at the path.

(5) Eq. (31) derived by the current analytical method is still valid in the plane perpendicular to the crack front and very close to the free surface, where the tri-axial stress constraint level is low.

This work was supported by the Fundamental Research Funds for the Central Universities (Grant No. 2014B1801).

1 R. J. Hartranft, and G. C. Sih, Int. J. Eng. Sci. 8, 711 (1970).
2 G. C. Sih, Int. J. Fract. Mech. 7, 39 (1971).

3 N. Levy, P. V. Marcal, and J. R. Rice, Nucl. Eng. Des. 17, 64 (1971).

4 J. P. Benthem, Int. J. Solids Struct. 13, 479 (1977).

5 T. Nakamura, and D. M. Parks, J. Appl. Mech. 55, 805 (1988).

6 W. Yang, and L. B. Freund, Int. J. Solids Struct. 21, 977 (1985).

7 R. Narasimhan, and A. J. Rosakis, J. Appl. Mech. 57, 607 (1990).

8 L. P. Pook, Eng. Fract. Mech. 48, 367 (1994).

9 N. Ranganathan, K. Jendoubi, and N. Merah, J. Eng. Mater. Technol. 116, 187 (1994).

10 X. M. Su, and C. T. Sun, Int. J. Fract. 82, 237 (1996).

11 S. W. Kwon, and C. T. Sun, Int. J. Fract. 104, 289 (2000).

12 A. Kotousov, Meccanica 39, 495 (2004).

13 C. She, and W. Guo, Int. J. Solids Struct. 44, 3021 (2007).

14 P. M. G. P. Moreira, S. D. Pastrama, and P. M. S. T. de Castro, Eng. Fract. Mech. 76, 2298 (2009).

15 R. A. Chaudhuri, Philos. Mag. 90, 2049 (2010).

16 J. C. Sobotka, and R. H. Dodds Jr., Eng. Fract. Mech. 78, 1182 (2011).

17 A. Kotousov, P. Lazzarin, F. Berto, and L. P. Pook, Eng. Fract. Mech. 108, 65 (2013).

18 S. M. A. Khan, N. Merah, and M. J. Adinoyi, Int. J. Solids Struct. 50, 1449 (2013).

19 R. C. O. Góes, J. T. P. Castro, and L. F. Martha, Int. J. Fatigue 62, 159 (2014).

20 Z. He, A. Kotousov, F. Berto, and R. Branco, Phys. Mesomech. 19, 6 (2016).

21 K. Kishimoto, S. Aoki, and M. Sakata, Eng. Fract. Mech. 13, 841 (1980).

22 C. F. Shih, B. Moran, and T. Nakamura, Int. J. Fract. 30, 79 (1986).

23 N. Omer, and Z. Yosibash, Int. J. Fract. 136, 1 (2005).

\section{Appendix}

Stain energy density $W$ (in dimensionless quantity) near the crack front may be written as:

$W=\frac{1}{2}\left(\sigma_{r} \varepsilon_{r}+\sigma_{\theta} \varepsilon_{\theta}+\sigma_{z} \varepsilon_{z}+2 \sigma_{r \theta} \varepsilon_{r \theta}\right)$.

Substituting the expressions of stress and strain (eqs. (22) and (25)) to eq. (a1), one may obtain

$$
\begin{aligned}
W= & \frac{K^{2}(z)}{64 \pi r}\left\{\left(5 \cos \frac{\theta}{2}-\cos \frac{3}{2} \theta\right)\left(\left(5-3 v-8 v T_{z}\right) \cos \frac{\theta}{2}-(1+v) \cos \frac{3}{2} \theta\right)\right. \\
& \left.+\left(3 \cos \frac{\theta}{2}+\cos \frac{3}{2} \theta\right)\left(\left(3-5 v-8 v T_{z}\right) \cos \frac{\theta}{2}+(1+v) \cos \frac{3}{2} \theta\right)+64 T_{z}\left(T_{z}-v\right) \cos ^{2} \frac{\theta}{2}+2(1+v)\left(\sin \frac{\theta}{2}+\sin \frac{3}{2} \theta\right)^{2}\right\} \\
= & \frac{K^{2}(z)}{64 \pi r}\left\{\left(34-30 v-128 v T_{z}+64\left(T_{z}\right)^{2}\right)\left(\cos \frac{\theta}{2}\right)^{2}+2(1+v)\left(\cos \frac{3}{2} \theta\right)^{2}-4(1+v) \cos \frac{3}{2} \theta \cos \frac{\theta}{2}+2(1+v)\left(\sin \frac{\theta}{2}+\sin \frac{3}{2} \theta\right)^{2}\right\} .
\end{aligned}
$$

Substituting eq. (a2) to eq. (27), one may have

$$
\begin{aligned}
\int_{\Gamma_{1}} W \mathrm{~d} y= & \int_{-\pi}^{\pi} W r_{1} \cos \theta \mathrm{d} \theta \\
= & \frac{K^{2}(z)}{64 \pi} \int_{-\pi}^{\pi}\left\{\left(34-30 v-128 v T_{z}+64\left(T_{z}\right)^{2}\right)\left(\cos \frac{1}{2} \theta\right)^{2}+2(1+v)\left(\cos \frac{3}{2} \theta\right)^{2}\right. \\
& \left.-4(1+v) \cos \frac{3}{2} \theta \cos \frac{\theta}{2}+2(1+v)\left(\sin \frac{\theta}{2}+\sin \frac{3}{2} \theta\right)^{2}\right\} \cos \theta \mathrm{d} \theta \\
= & \frac{K^{2}(z)}{4}\left(2\left(T_{z}\right)^{2}-4 v T_{z}-v+1\right) .
\end{aligned}
$$

According to eq. (29), one may have 


$$
\begin{aligned}
-\int_{\Gamma_{1}} \sigma_{i j} n_{j} u_{i, x} \mathrm{~d} s & =-\int_{-\pi}^{\pi}\left(\sigma_{x} n_{x} u_{x, x}+\sigma_{x y} n_{y} u_{x, x}+\sigma_{x y} n_{x} u_{y, x}+\sigma_{y} n_{y} u_{y, x}\right) r_{1} \mathrm{~d} \theta \\
& =-\int_{-\pi}^{\pi}\left(T_{x} u_{x, x}+T_{y} u_{y, x}\right) r_{1} \mathrm{~d} \theta .
\end{aligned}
$$

Here, $T_{x}$ and $T_{y}$ represent the traction along the $x$ and $y$ axes separately at the path $\Gamma_{1}$, see Figure 9 . At the path $\Gamma_{1}, T_{x}$ and $T_{y}$ may be expressed by $\sigma_{r}$ and $\sigma_{\theta}$ as:

$T_{x}=\sigma_{r} \cos \theta-\sigma_{r \theta} \sin \theta$,

$T_{y}=\sigma_{r} \sin \theta+\sigma_{r \theta} \cos \theta$.

Similarly, at the path $\Gamma_{1}$, displacement components in rectangular coordinates may be expressed by corresponding components in cylindrical coordinates, i.e.,

$u_{x}=u_{r} \cos \theta-u_{\theta} \sin \theta$

$u_{y}=u_{r} \sin \theta+u_{\theta} \cos \theta$.

Noting $\frac{\partial \theta}{\partial x}=-\frac{\sin \theta}{r}$ and $\frac{\partial r}{\partial x}=\cos \theta$, one may get

$\frac{\partial u_{x}}{\partial x}=\frac{\partial u_{x}}{\partial \theta} \frac{\partial \theta}{\partial x}+\frac{\partial u_{x}}{\partial r} \frac{\partial r}{\partial x}=-\frac{\sin \theta}{r} \frac{\partial u_{x}}{\partial \theta}+\cos \theta \frac{\partial u_{x}}{\partial r}$,

$\frac{\partial u_{y}}{\partial x}=\frac{\partial u_{y}}{\partial \theta} \frac{\partial \theta}{\partial x}+\frac{\partial u_{y}}{\partial r} \frac{\partial r}{\partial x}=-\frac{\sin \theta}{r} \frac{\partial u_{y}}{\partial \theta}+\cos \theta \frac{\partial u_{y}}{\partial r}$.

Substituting eq. (a6) to (a7), one may get

$\frac{\partial u_{x}}{\partial x}=-\frac{\sin \theta}{r}\left(\left(\left(u_{r}\right)^{\prime}-u_{\theta}\right) \cos \theta-\left(\left(u_{\theta}\right)+u_{r}\right) \sin \theta\right)+\cos \theta\left(\left(u_{r}\right)^{\prime} \cos \theta-\left(u_{\theta}\right)^{\prime} \sin \theta\right)$,

$\frac{\partial u_{y}}{\partial x}=-\frac{\sin \theta}{r}\left(\left(u_{r}+\left(u_{\theta}\right)\right) \cos \theta+\left(\left(u_{r}\right)-u_{\theta}\right) \sin \theta\right)+\cos \theta\left(\left(u_{r}\right)^{\prime} \sin \theta+\left(u_{\theta}\right)^{\prime} \cos \theta\right)$.

Noting eqs. (a5) and (a8), one may have

$$
\begin{aligned}
\sigma_{i j} n_{j} u_{i, x}= & T_{x} u_{x, x}+T_{y} u_{y, x} \\
= & \left(\sigma_{r} \cos \theta-\sigma_{r \theta} \sin \theta\right)\left(-\frac{\sin \theta}{r}\left(\left(\left(u_{r}\right)^{-}-u_{\theta}\right) \cos \theta-\left(\left(u_{\theta}\right)^{\prime}+u_{r}\right) \sin \theta\right)+\cos \theta\left(\left(u_{r}\right)^{\prime} \cos \theta-\left(u_{\theta}\right)^{\prime} \sin \theta\right)\right) \\
& +\left(\sigma_{r} \sin \theta+\sigma_{r \theta} \cos \theta\right)\left(-\frac{\sin \theta}{r}\left(\left(u_{r}+\left(u_{\theta}\right)^{\prime}\right) \cos \theta+\left(\left(u_{r}\right)^{\prime}-u_{\theta}\right) \sin \theta\right)+\cos \theta\left(\left(u_{r}\right)^{\prime} \sin \theta+\left(u_{\theta}\right)^{\prime} \cos \theta\right)\right) \\
& =\sigma_{r}\left(-\frac{\sin \theta}{r}\left(\left(u_{r}\right)^{\prime}-\left(u_{\theta}\right)\right)+\left(u_{r}\right)^{\prime} \cos \theta\right)+\sigma_{r \theta}\left(-\frac{\sin \theta}{r}\left(\left(u_{\theta}\right)^{\prime}+u_{r}\right)+\cos \theta\left(u_{\theta}\right)^{\prime}\right) .
\end{aligned}
$$

Substituting eqs. (22) and (30) to the first and second terms in the last line in eq. (a9), one may have

$$
\begin{aligned}
& \sigma_{r}\left(-\frac{\sin \theta}{r}\left(\left(u_{r}\right)^{\prime}-\left(u_{\theta}\right)\right)+\left(u_{r}\right)^{\prime} \cos \theta\right)=\frac{K^{2}(z)\left(5 \cos \frac{1}{2} \theta-\cos \frac{3}{2} \theta\right)}{32 \pi r} \\
& \times\left\{-\left(9+v-8 v T_{z}\right) \sin \frac{1}{2} \theta \sin \theta-(1+v) \sin \frac{3}{2} \theta \sin \theta+\left(5-3 v-8 v T_{z}\right) \cos \frac{1}{2} \theta \cos \theta-(1+v) \cos \frac{3}{2} \theta \cos \theta\right\} .
\end{aligned}
$$

and

$$
\begin{aligned}
& \sigma_{r \theta}\left(-\frac{\sin \theta}{r}\left(\left(u_{\theta}\right)^{\prime}+u_{r}\right)+\cos \theta\left(u_{\theta}\right)^{\prime}\right)=\frac{K^{2}(z)\left(\sin \frac{1}{2} \theta+\sin \frac{3}{2} \theta\right)}{32 \pi r} \\
& \times\left\{-\left(3-5 v-8 v T_{z}\right) \cos \frac{1}{2} \theta \sin \theta-(1+v) \cos \frac{3}{2} \theta \sin \theta-\left(7-v-8 v T_{z}\right) \sin \frac{1}{2} \theta \cos \theta+(1+v) \sin \frac{3}{2} \theta \cos \theta\right\} .
\end{aligned}
$$

Substituting eqs. (a10) and (a11) to eq. (a9), one may have 
D. Yi, et al. Sci. China-Phys. Mech. Astron. June (2018) Vol.61 No.6

064611-12

$$
\begin{aligned}
\sigma_{i j} n_{j} u_{i, x}= & T_{x} u_{x, x}+T_{y} u_{y, x} \\
= & \sigma_{r}\left(-\frac{\sin \theta}{r}\left(\left(u_{r}\right)^{-}-\left(u_{\theta}\right)\right)+\left(u_{r}\right)^{\prime} \cos \theta\right)+\sigma_{r \theta}\left(-\frac{\sin \theta}{r}\left(\left(u_{\theta}\right)+u_{r}\right)+\cos \theta\left(u_{\theta}\right)^{\prime}\right) \\
= & \frac{K^{2}(z)\left(5 \cos \frac{1}{2} \theta-\cos \frac{3}{2} \theta\right)}{32 \pi r}\left\{-\left(9+v-8 v T_{z}\right) \sin \frac{1}{2} \theta \sin \theta-(1+v) \sin \frac{3}{2} \theta \sin \theta\right. \\
& \left.+\left(5-3 v-8 v T_{z}\right) \cos \frac{1}{2} \theta \cos \theta-(1+v) \cos \frac{3}{2} \theta \cos \theta\right\}+\frac{K^{2}(z)\left(\sin \frac{1}{2} \theta+\sin \frac{3}{2} \theta\right)}{32 \pi r} \\
& \times\left\{-\left(3-5 v-8 v T_{z}\right) \cos \frac{1}{2} \theta \sin \theta-(1+v) \cos \frac{3}{2} \theta \sin \theta-\left(7-v-8 v T_{z}\right) \sin \frac{1}{2} \theta \cos \theta+(1+v) \sin \frac{3}{2} \theta \cos \theta\right\} .
\end{aligned}
$$

Substituting eq. (a12) to (a4), one may have

$$
\begin{aligned}
-\int_{\Gamma_{1}} \sigma_{i j} n_{j} u_{i, x} \mathrm{~d} s= & -\frac{K^{2}(z)}{32 \pi} \int_{-\pi}^{\pi}\left(5 \cos \frac{1}{2} \theta-\cos \frac{3}{2} \theta\right)\left\{-\left(9+v-8 v T_{z}\right) \sin \frac{1}{2} \theta \sin \theta-(1+v) \sin \frac{3}{2} \theta \sin \theta\right. \\
& \left.+\left(5-3 v-8 v T_{z}\right) \cos \frac{1}{2} \theta \cos \theta-(1+v) \cos \frac{3}{2} \theta \cos \theta\right\} \mathrm{d} \theta \\
& -\frac{K^{2}(z)}{32 \pi} \int_{-\pi}^{\pi}\left(\sin \frac{1}{2} \theta+\sin \frac{3}{2} \theta\right)\left\{-\left(3-5 v-8 v T_{z}\right) \cos \frac{1}{2} \theta \sin \theta-(1+v) \cos \frac{3}{2} \theta \sin \theta\right. \\
& \left.-\left(7-v-8 v T_{z}\right) \sin \frac{1}{2} \theta \cos \theta+(1+v) \sin \frac{3}{2} \theta \cos \theta\right\} \mathrm{d} \theta \\
= & -\frac{K^{2}}{4}\left(2 v T_{z}-v-3\right) .
\end{aligned}
$$

